\title{
ASSOCIAÇÕES ENTRE POLIMORFISMOS GENÉTICOS DA ÁLCOOL: DESIDROGENASE E O TRANSTORNO POR USO DE ÁLCOOL
}

\author{
ASSOCIATIONS BETWEEN GENETIC POLYMORPHISMS \\ OF ALCOHOL: DEHYDROGENASE AND ALCOHOL USE \\ DISORDER
}

\author{
Jonas Michel Wolf ${ }^{1}$, Daniel Simon', Vagner Ricardo Lunge ${ }^{1}$
}

\section{RESUMO}

Clin Biomed Res. 2019;39(4):322-332

1 Universidade Luterana do Brasil (Ulbra). Canoas, Rio Grande do Sul, Brasil.

Autor correspondente: Jonas Michel Wolf jonasmwolf@gmail.com Universidade Luterana do Brasil (Ulbra).

Avenida Farroupilha, 8001. 92425-900, Canoas, RS, Brasil.
O transtorno por uso de álcool (TUA) é influenciado pela genética, principalmente na metabolização do etanol. Os genes da álcool desidrogenase (ADH1BIADH1C), enzima que transforma o etanol, apresentam SNPs (single nucleotide polymorphisms) que resultam em isoenzimas com diferentes taxas catalíticas. Estudos demonstraram que os SNPs Arg48His, Arg370Cys, Arg272GIn e lle350Val contribuem para o TUA. Este artigo revisou os estudos que investigaram SNPs em ADH1B (Arg48His/Arg370Cys) e $A D H 1 C$ (Arg272GIn/lle350Val), bem como avaliou as variações nas frequências alélicas desses genes e a influência no TUA nas diferentes populações no mundo. As frequências alélicas dos polimorfismos foram comparadas pelos testes qui-quadrado de Pearson e exato de Fisher $(p<0,05)$. O SNP Arg48His confere proteção para o TUA em euroamericanos, latino-americanos, europeus, brasileiros, asiáticos e australianos. O SNP Arg370Cys confere proteção para o TUA em afrodescendentes. Os SNPs Arg272GIn e lle350Val predispõem o TUA principalmente em europeus. Os SNPs Arg48His, Arg370Cys e Arg272GIn/lle350Val foram mais frequentes em amostras de leste-asiáticos $(69,7 \%)$, africanos $(19,1 \%)$ e europeus $(40,5 \%)$, respectivamente $(p<0,01)$. Os diferentes alelos dos genes $A D H 1 B I A D H 1 C$ devido a SNPs têm uma importante contribuição no TUA. As frequências desses alelos variam conforme a população, resultando em diferentes efeitos no TUA.

Palavras-chave: Transtorno por uso de álcool; álcool desidrogenase; SNPs

\section{ABSTRACT}

Alcohol use disorder (AUD) is influenced by genetics, especially in the metabolism of ethanol. The ethanol dehydrogenase genes (ADH1BIADH1C), which convert ethanol, have single nucleotide polymorphisms (SNPs) that result in isoenzymes with different catalytic rates. Studies have shown that the Arg48His, Arg370Cys, Arg272GIn, and Ile350Val SNPs contribute to AUD. This article reviewed the studies that investigated SNPs in ADH1B (Arg48His/Arg370Cys) and ADH1C (Arg272Gln/lle350Val) and evaluated variations in the allele frequencies of these genes and their influence on AUD in different populations worldwide. The allele frequencies of the polymorphisms were compared by Pearson's chi-square and Fisher's exact tests $(p<0.05)$. The Arg48His SNP provides protection against AUD in Euro-Americans, Latin Americans, Europeans, Brazilians, Asians, and Australians. The Arg370Cys SNP provides protection against AUD in Afro-descendants. The Arg272GIn and lle350Val SNPs predispose to AUD mainly in Europeans. The Arg48His, Arg370Cys, and Arg272GIn/lle350Val SNPs were more frequent in East Asians (69.7\%), Africans (19.1\%), and Europeans $(40.5 \%)$, respectively $(p<0.01)$. The different alleles of the $A D H 1 B I A D H 1 C$ genes due to SNPs make an important contribution to AUD. The frequencies of these alleles vary among different populations, resulting in different effects on AUD.

Keywords: Alcohol use disorder; alcohol dehydrogenase; single nucleotide polymorphism 
O consumo de substâncias alcoólicas ocorre em todas as regiões do mundo ${ }^{1}$. Existem registros de utilização destas substâncias desde o início da civilização humana ${ }^{2}$. Atualmente, este consumo abrange aproximadamente $38,0 \%$ da população mundial ${ }^{3}$. Além disso, se estima que cerca de $16,0 \%$ da população consuma álcool de forma abusiva e consequentemente podem evoluir para o transtorno por uso de álcool (TUA) $)^{3,4}$.

Especificamente, no Brasil, em uma pesquisa que avaliou mais de 3000 pessoas, foi detectado que $11 \%$ dos indivíduos bebiam álcool diariamente e $28 \%$ de três a quatro vezes por semana ${ }^{5}$. Em um levantamento incluindo 108 cidades brasileiras, foi observada que $69 \%$ dos indivíduos já fizeram uso de álcool na vida. A ingestão periódica de álcool (mínimo de 3 a 4 vezes por semana, incluindo aqueles que bebem todos os dias), foi contabilizada em $9 \%$ dos homens e $2 \%$ das mulheres ${ }^{6}$. Dados da Organização Mundial da Saúde (OMS) demonstram que a prevalência do TUA no Brasil é de $6 \%$. Contudo, quando estratificada por sexo representa percentuais de $8 \%$ para homens e $3 \%$ para mulheres ${ }^{7}$. Além disso, o consumo frequente de álcool (de uma a quatro vezes por semana) varia entre as regiões do país, sendo observados os seguintes percentuais: $25 \%$ no Sul, $21 \%$ no Nordeste, $18 \%$ no Sudeste, $18 \%$ no Centro-Oeste e $10 \%$ no Norte $^{5}$. Já o consumo muito frequente (beber todos os dias), foi observado em $11 \%$ no Sul, $6 \%$ no Sudeste, $6 \%$ no Centro-Oeste, $4 \%$ no Norte e $3 \%$ no Nordeste 5 .

O TUA é multifatorial e envolve aspectos genéticos e ambientais ${ }^{8,9}$. Alguns genes relacionados aos mecanismos de metabolização do álcool possuem alelos associados ao TUA, e, por conseguinte, têm sido o foco de pesquisas científicas em populações de diferentes etnias ${ }^{10-13}$. Entre estes, sete estão localizados em um grupo de genes no cromossomo 4 e codificam as subunidades enzimáticas da álcool desidrogenase $(A D H)^{9,14-20}$. Esta enzima atua na mucosa gástrica e majoritariamente no tecido hepático, convertendo o álcool em acetaldeído, sendo este um processo crucial para a eliminação do álcool do organismo ${ }^{12-15}$. As enzimas codificadas pelos genes pertencentes à classe I da ADH (ADH1A, ADH1B e $A D H 1 C$ ) apresentam maiores atividades e são as principais responsáveis pela metabolização hepática do álcool. Portanto, variantes nestes genes, principalmente em $A D H 1 B$ e $A D H 1 C$, são amplamente estudadas no contexto genético do TUA $^{9,12,17,20 .}$.

Polimorfismos de nucleotídeo único (SNPs, do inglês single nucleotide polymorphisms) nos genes que codificam a $A D H$, em especial os que resultam em troca de aminoácidos, são responsáveis pela síntese de enzimas com diferentes taxas de metabolização do álcool ${ }^{14,20}$. Especificamente, dois SNPs no gene $A D H 1 B$ (Arg48His, C > T, rs1229984; Arg370Cys, C > T, rs2066702) permitem a metabolização do álcool em uma velocidade aproximadamente 80 vezes maior do que a forma selvagem do gene $A D H 1 B$, resultando em um aumento dos níveis de acetaldeído no organismo, o que causa proteção contra o TUA $^{14-16,20,21}$. Isso ocorre, pois o acetaldeído causa uma sintomatologia desconfortável, principalmente náuseas, fazendo com que o indivíduo não consuma grandes quantidades de substâncias alcoólicas ${ }^{8-13,20,21}$. Quanto maior a concentração sérica de acetaldeído, maiores serão os efeitos adversos e menor a probabilidade de continuidade do consumo de álcool ${ }^{8,10,11,15-21}$. O gene $A D H 1 C$ também apresenta SNPs associados ao desenvolvimento do TUA ${ }^{8,14,16}$. Arg272GIn (G > A, rs1693482) e lle350Val $(A>G$, rs698), por exemplo, retardam a metabolização do álcool e conferem risco de TUA ${ }^{15}$.

As frequências dos SNPs variam consideravelmente entre as populações mundiais. O polimorfismo de proteção, Arg48His, é frequentemente encontrado em asiáticos do Leste e incomum em outras populações, como caucasianos, ameríndios e afrodescendentes ${ }^{14-16,20}$. Já, o polimorfismo Arg370Cys está presente em aproximadamente $20 \%$ dos afrodescendentes e sua presença em outras etnias é rara ${ }^{13,14}$. Adicionalmente, os polimorfismos do gene $A D H 1 C$, que conferem risco ao TUA, são mais frequentes $(\approx 40 \%)$ em populações de eurodescendentes ${ }^{13-16}$. A Figura 1 apresenta esses quatro principais SNPs dos genes $A D H 1 B$ e $A D H 1 C$.

Estudos que avaliaram possíveis associações desses SNPs de genes de metabolização do álcool e o TUA ainda são escassos na América Latina, principalmente no Brasil. O presente estudo teve por objetivo realizar uma ampla revisão da literatura sobre os quatro principais SNPs nos genes da ADH (Arg48His, Arg370Cys, Arg272GIn e lle350Val) e sua relação com o TUA, tanto no Brasil como no mundo. $O$ estudo da relação entre a genética do metabolismo do álcool e o TUA é essencial para um melhor conhecimento deste transtorno. Nesse sentido, as frequências alélicas dos polimorfismos nos genes $A D H 1 B$ e $A D H 1 C$ foram extraídas da base de dados Ensembl Genome Browser 92 e avaliadas estatisticamente, visando a compreensão da variabilidade genética em diferentes regiões do mundo e como este processo pode estar associado ao TUA. 
Genes de classe I da ADH

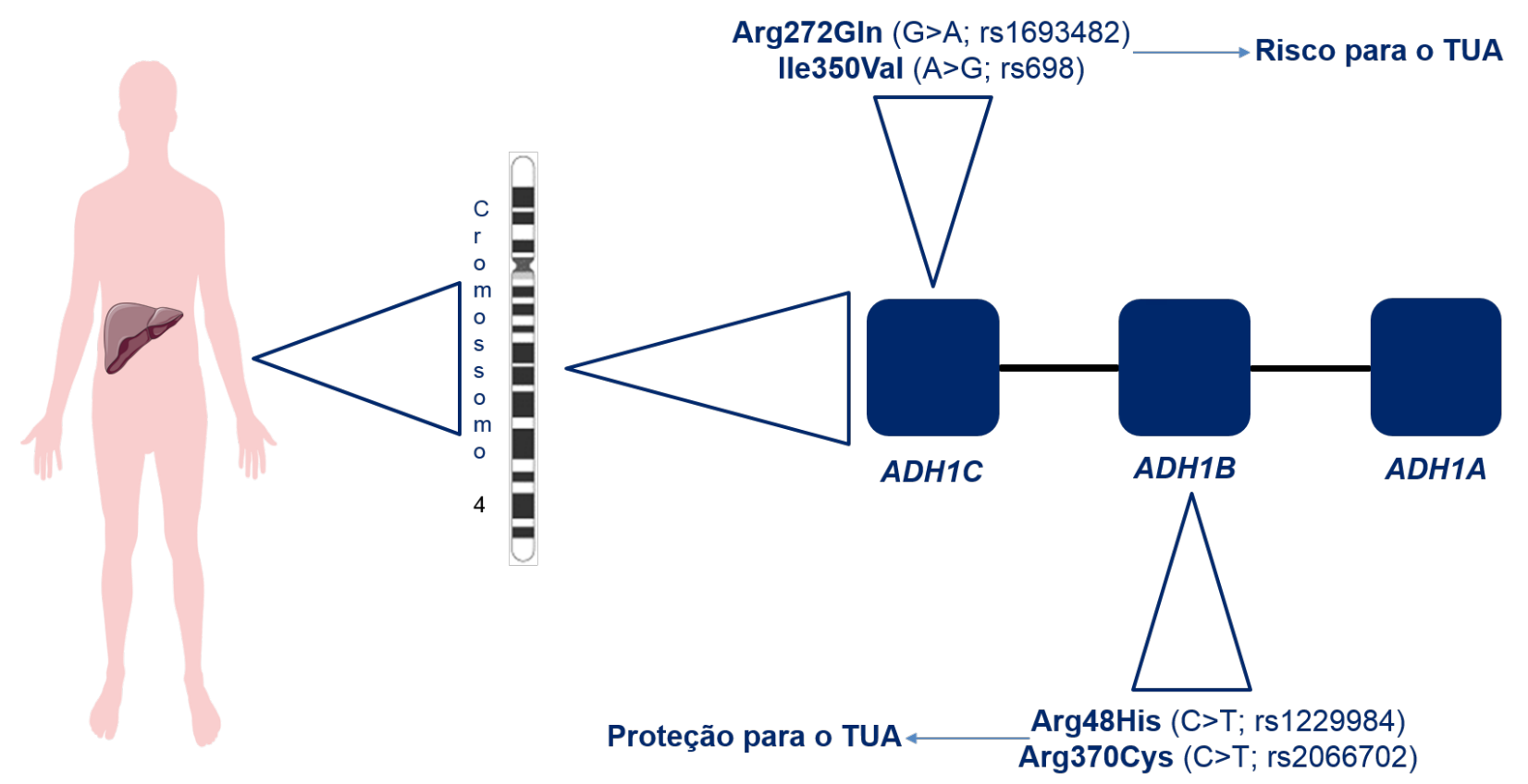

Figura 1: Representação gráfica dos genes $A D H$ de classe I localizados no cromossomo 4 e os SNPs mais estudados nos genes $A D H 1 C$ (Arg272GIn e lle350Val) e ADH1B (Arg48His e Arg370Cys). Estes polimorfismos atuam de forma determinante no metabolismo hepático do álcool. Fonte: Figura construída pelos autores do presente estudo.

\section{METODOLOGIA}

\section{Revisão da literatura}

Os artigos científicos foram selecionados a partir de buscas nos seguintes bancos de dados de publicações: US National Library of Medicine National Institutes of Health (NCBI / PUBMED), Google Scholar, Scientific Eletronic Library Online (SciELO), Science Direct, Medical Literature Analysis and Retrieval System Online (MEDLINE), Literatura Latino-Americana e do Caribe em Ciências da Saúde (LILACS) e Scopus. As palavras-chave utilizadas foram: "alcohol metabolism", "genetics and alcohol use disorder", "genetics polymorphisms and alcohol use disorder", "alcohol dehydrogenases", "alcohol dehydrogenases polymorphisms", "studies of associations between genetic polymorphisms and alcohol use disorder" e "genome-wide association study of alcohol dehydrogenase". As pesquisas foram realizadas focando artigos que contemplassem o escopo desta revisão. Os artigos que apresentaram os delineamentos de GWAS (Genome-wide association study) e caso-controle, que apresentaram um número amostral mínimo de 50 indivíduos e que utilizaram como métodos de genotipagem a discriminação alélica foram priorizados nesta revisão. O período de publicação do material selecionado foi de 1996 a 2018. Relatos de casos, correspondências, comentários e artigos completos que não apresentavam relações com o escopo da presente revisão foram excluídos.

\section{Coleta de dados dos polimorfismos nos genes ADH1B e ADH1C}

As coletas dos dados referentes as frequências dos polimorfismos nos genes $A D H 1 B$ e $A D H 1 C$ foram realizadas a partir do banco de dados Ensembl Genome Browser 92 (https://www.ensembl.org/index. html) com os seguintes descritores: rs1229984, rs2066702, rs1693482 e rs698. Essas frequências foram extraídas de diferentes regiões do mundo, como o continente africano, o europeu, o americano, o Sul da Ásia e o Leste da Ásia. Após este processo, os dados foram avaliados estatisticamente.

\section{Análises estatísticas}

Os dados foram compilados e analisados com o uso do software SPSS $尺$ (IL Statistical Package for the Social Sciences, 23.0 version, Chicago). A avaliação de diferenças estatísticas entre os continentes com relação as frequências alélicas dos polimorfismos nos genes ADH1B (Arg48His e Arg370Cys) e ADH1C (Arg272GIn e lle350Val) foi realizada pelos testes qui-quadrado de Pearson e exato de Fisher, conforme apropriado. Todas as estimativas foram bilaterais 
com nível de significância pré-estabelecido para o erro alfa de $5 \%(p<0,05)$.

\section{RESULTADOS}

\section{Associações dos polimorfismos nos genes ADH com O TUA}

Associações dos genes de $A D H$ com TUA foram observadas em amostras independentes, incluindo afro-americanos ${ }^{10,22-26}$, euroamericanos ${ }^{23-25} \mathrm{e}^{2}$ asiáticos $^{26}$. No estudo de Gelernter et al. (2014) ${ }^{23}$, que consistiu de um GWAS (genome-wide association study), seguido de uma avaliação caso-controle com 16.087 amostras, foram relatados resultados significativos, principalmente referentes aos polimorfismos Arg48His em euroamericanos $\left(p=1,17 \times 10^{-31}\right)$ e Arg370Cys em afro-americanos $\left(p=6,33 \times 10^{-17}\right)$. Estas associações foram replicadas, considerando os escores dos critérios do DSM (do inglês, Diagnostic and Statistical Manual of Mental Disorders) IV e V ${ }^{24}$. Além disso, estes polimorfismos foram analisados e 0 TUA foi associado com Arg48His em euroamericanos $\left(p=5,96 \times 10^{-15}\right)$ e Arg370Cys em afro-americanos $\left(p=2,50 \times 10^{-10}\right)^{25}$. As Tabelas 1, 2, 3 e 4 apresentam os principais artigos publicados e os respectivos resultados sobre o tema.

Tabela 1: Estudos que detectaram associações entre o polimorfismo Arg48His do gene ADH1B e a proteção para o TUA.

\begin{tabular}{|c|c|c|c|c|}
\hline Etnias / Estudos & Delineamento & Desfecho avaliado & $\begin{array}{c}\mathbf{N}^{0} \text { de } \\
\text { amostras }\end{array}$ & Valor de $p$ \\
\hline \multicolumn{5}{|l|}{ Euroamericanos } \\
\hline Luo et al. $(2006)^{27}$ & Caso-controle & Dependência alcoólica & 651 & $<0,01$ \\
\hline Tolstrup et al. $(2008)^{28}$ & Caso-controle & Dependência alcoólica & 9222 & $<0,05$ \\
\hline Sherva et al. $(2009)^{29}$ & GWAS* & Consumo abusivo & 1588 & $<0,01$ \\
\hline Bierut et al. $(2012)^{30}$ & Caso-controle & Dependência alcoólica & 1800 & $6,6 \times 10^{-6}$ \\
\hline Gelernter et al. $(2014)^{23}$ & GWAS & Dependência alcoólica & 10654 & $\underset{31}{1,17 \times 10^{-}}$ \\
\hline Hart et al. $(2015)^{24}$ & GWAS & Dependência alcoólica & 2368 & $5,2 \times 10^{-12}$ \\
\hline Xu et al. $(2015)^{25}$ & GWAS & Dependência alcoólica & 5064 & $5,6 \times 10^{-15}$ \\
\hline \multicolumn{5}{|l|}{ Mexicanos-americanos } \\
\hline Ehlers et al. (2012) ${ }^{31}$ & Caso-controle & Dependência alcoólica & 439 & $<0,01$ \\
\hline \multicolumn{5}{|l|}{ Europeus } \\
\hline Borras et al. $(2000)^{32}$ & Caso-controle & Dependência alcoólica & 876 & $<0,01$ \\
\hline Cichoz-Lach et al. $(2010)^{33}$ & Caso-controle & Dependência alcoólica & 376 & $<0,01$ \\
\hline Linneberg et al. $(2010)^{34}$ & Caso-controle & Reação fisiológica ao álcool & 8000 & $<0,05$ \\
\hline Tóth et al. $(2010)^{35}$ & Caso-controle & Dependência alcoólica & 1030 & $<0,01$ \\
\hline Hubacek et al. $(2012)^{36}$ & Caso-controle & Dependência alcoólica & 6497 & $<0,05$ \\
\hline Way et al. $(2015)^{37}$ & Caso-controle & Dependência alcoólica & 2103 & $<0,01$ \\
\hline \multicolumn{5}{|l|}{ Brasileiros } \\
\hline Carvalho et al. $(2013)^{38}$ & Caso-controle & Dependência alcoólica & 345 & 0,03 \\
\hline Wolf et al. $(2017)^{39}$ & Caso-controle & Consumo abusivo & 365 & 0,02 \\
\hline \multicolumn{5}{|l|}{ Asiáticos } \\
\hline Chen et al. $(1996)^{40}$ & Caso-controle & Dependência alcoólica & 109 & $<0,01$ \\
\hline Tanaka et al. $(1996)^{41}$ & Caso-controle & Dependência alcoólica & 156 & $<0,01$ \\
\hline Li et al. $(2011)^{42}$ & Meta-análise & Dependência alcoólica & 19155 & $1 \times 10^{-36}$ \\
\hline Meyers et al. $(2013)^{43}$ & Caso-controle & Consumo abusivo & 1349 & $<0,01$ \\
\hline Kilcoyne et al. $(2014)^{44}$ & Caso-controle & Consumo abusivo & 1130 & $<0,01$ \\
\hline Al-Tu'ma et al. $(2015)^{45}$ & Caso-controle & Dependência alcoólica & 120 & $<0,05$ \\
\hline \multicolumn{5}{|l|}{ Australianos } \\
\hline Macgregor et al. (2009) & GWAS & Dependência alcoólica & 4597 & $<0,01$ \\
\hline
\end{tabular}


Wolf et al.

Tabela 2: Estudos que detectaram associações entre o polimorfismo Arg370Cys do gene $A D H 1 B$ e a proteção ao TUA

\begin{tabular}{|c|c|c|c|c|}
\hline Etnias / Estudos & Delineamento & Desfecho avaliado & $\mathrm{N}^{\circ}$ de amostras & Valor de $p$ \\
\hline \multicolumn{5}{|l|}{ Afrodescendentes } \\
\hline Ehlers et al. $(2003)^{47}$ & Clínico & Reação fisiológica ao álcool & 66 & $<0,05$ \\
\hline Edenberg et al. $(2006)^{48}$ & GWAS & Reação fisiológica ao álcool & 279 & $<0,05$ \\
\hline Luo et al. $(2006)^{27}$ & Caso-controle & Reação fisiológica ao álcool & 150 & $<0,01$ \\
\hline McCarthy et al. (2010) $)^{49}$ & Clínico & Reação fisiológica ao álcool & 91 & $<0,05$ \\
\hline Gelernter et al. $(2014)^{23}$ & GWAS & Dependência alcoólica & 5430 & $6,33 \times 10^{-17}$ \\
\hline Hart et al. $(2015)^{24}$ & GWAS & Dependência alcoólica & 3301 & $1,4 \times 10^{-9}$ \\
\hline Xu et al. $(2015)^{25}$ & GWAS & Consumo abusivo & 4491 & $2,50 \times 10^{-10}$ \\
\hline \multicolumn{5}{|l|}{ Nativo-americanos } \\
\hline Wall et al. $(2003)^{50}$ & Caso-controle & Dependência alcoólica & 340 & $<0,01$ \\
\hline Gizer et al. $(2011)^{51}$ & GWAS & Reação fisiológica ao álcool & 586 & $<0,01$ \\
\hline Ehlers et al. $(2012)^{31}$ & Caso-controle & Dependência alcoólica & 792 & 0,03 \\
\hline \multicolumn{5}{|l|}{ Afro-caribenhos } \\
\hline Montane-Jaime et al. $(2014)^{52}$ & Clínico & Reação fisiológica ao álcool & 112 & $<0,05$ \\
\hline
\end{tabular}

GWAS: Genome-wide association study

Tabela 3: Estudos que detectaram associações entre o polimorfismo Arg272Gln do gene $A D H 1 C$ e o risco ao TUA

\begin{tabular}{|c|c|c|c|c|}
\hline Etnias / Estudos & Delineamento & Desfecho avaliado & $\mathrm{N}^{\circ}$ de amostras & Valor de $p$ \\
\hline \multicolumn{5}{|l|}{ Europeus } \\
\hline Kuo et al. $(2008)^{53}$ & Caso-controle & Dependência alcoólica & 1105 & 0,04 \\
\hline *Martínez et al. (2010)54 & Clínico & Reação fisiológica ao álcool & 250 & $<0,01$ \\
\hline Tóth et al. $(2011)^{55}$ & Caso-controle & Dependência alcoólica & 907 & 0,03 \\
\hline Kortunay et al. (2012) $)^{56}$ & Caso-controle & Dependência alcoólica & 190 & $<0,01$ \\
\hline Way et al. $(2015)^{37}$ & Caso-controle & Dependência alcoólica & 2103 & $<0,05$ \\
\hline \multicolumn{5}{|l|}{ Australianos } \\
\hline Macgregor et al. (2009) & GWAS & $\begin{array}{l}\text { Reação fisiológica ao álcool/ } \\
\text { consumo abusivo }\end{array}$ & 4597 & $<0,01$ \\
\hline
\end{tabular}

GWAS: Genome-wide association study

Tabela 4: Estudos que detectaram associações entre o polimorfismo lle350Val do gene ADH1C e o risco ao TUA

\begin{tabular}{|c|c|c|c|c|}
\hline Etnias / Estudos & Delineamento & Desfecho avaliado & $\mathrm{N}^{0}$ de amostras & Valor de $p$ \\
\hline \multicolumn{5}{|l|}{ Europeus } \\
\hline Borras et al. (2000) $)^{32}$ & Caso-controle & Dependência alcoólica & 876 & $<0,05$ \\
\hline Tolstrup et al. $(2008)^{28}$ & Caso-controle & Dependência alcoólica & 9222 & $<0,05$ \\
\hline Cichoz-Lach et al. $(2010)^{33}$ & Caso-controle & Dependência alcoólica & 376 & $<0,01$ \\
\hline${ }^{*}$ Martínez et al. $(2010)^{54}$ & Clínico & $\begin{array}{l}\text { Reação fisiológica ao álcool/ } \\
\text { consumo abusivo }\end{array}$ & 250 & $<0,01$ \\
\hline Tóth et al. $(2011)^{55}$ & Caso-controle & Dependência alcoólica & 907 & 0,01 \\
\hline Kortunay et al. (2012) $)^{56}$ & Caso-controle & Dependência alcoólica & 190 & $<0,01$ \\
\hline Ayhan et al. $(2015)^{59}$ & Caso-controle & Dependência alcoólica & 235 & $<0,05$ \\
\hline Way et al. $(2015)^{37}$ & Caso-controle & Dependência alcoólica & 2103 & $<0,05$ \\
\hline \multicolumn{5}{|l|}{ Asiáticos } \\
\hline Chen et al. $(1996)^{60}$ & Caso-controle & Dependência alcoólica & 109 & $<0,05$ \\
\hline Meyers et al. $(2013)^{43}$ & Caso-controle & Consumo abusivo & 1349 & $<0,01$ \\
\hline Bjerregaard et al. $(2014)^{61}$ & Caso-controle & Consumo abusivo & 4162 & $<0,05$ \\
\hline \multicolumn{5}{|l|}{ Afro-caribenhos } \\
\hline Montane-Jaime et al. $(2006)^{62}$ & Caso-controle & Dependência alcoólica & 253 & $<0,01$ \\
\hline${ }^{*}$ Montane-Jaime et al. $(2014)^{52}$ & Clínico & $\begin{array}{l}\text { Reação fisiológica ao álcool/ } \\
\text { consumo abusivo }\end{array}$ & 112 & $<0,05$ \\
\hline
\end{tabular}

*Associação do polimorfismo com a intolerância aos efeitos do álcool 
As relações entre os polimorfismos no gene $A D H 1 B$ (Arg48His e Arg370Cys) e o TUA tem sido frequentemente investigadas ${ }^{23-28}$. Especificamente o polimorfismo Arg48His demonstrou proteção para o TUAem amostras da população geral de euroamericanos, latino-americanos, europeus, brasileiros, asiáticos e australianos (Tabela 1). Já a contribuição do polimorfismo Arg370Cys para a proteção do TUA aparentemente possui relações exclusivas com a ascendência africana. Este aspecto já foi evidenciado por investigações conduzidas com afrodescendentes, nativos-americanos e afrocaribenhos (Tabela 2). Contudo, até o momento nenhum estudo disponível na literatura relatou associações deste polimorfismo e TUA em indivíduos com ascendência europeia, euroamericana ou sul-americana.

Além dos dois polimorfismos acima citados no gene $A D H 1 B$, estudos prévios também analisaram os dois SNPs mais frequentes no gene $A D H 1 C$. A relação do polimorfismo Arg272GIn com o risco do TUA foi verificada apenas em estudos conduzidos com amostras europeias e da Austrália (Tabela 3). A literatura não contempla o conhecimento deste polimorfismo em amostras de outras regiões do mundo. Estudos de Rebello et al. (2011) $)^{57}$ e Wolf et al. (2017) ${ }^{39}$ conduzidos no Brasil, e de Méndez e Rey, $(2015)^{58}$ realizado na Colômbia, avaliaram a potencial contribuição do polimorfismo lle350Val em relação ao TUA, e não detectaram associação significativa. Este polimorfismo já foi relacionado com o consumo de álcool em amostras de europeus (Tabela 4). Além disso, a intolerância biológica frente aos efeitos do álcool, na presença do polimorfismo lle350Val já foi verificada em afro-caribenhos (Tabela 4). Aparentemente, não há estudos que associaram este polimorfismo em amostras de sul-americanos, norte-americanos e de africanos.

\section{Análise comparativa das frequências dos poli- morfismos nos genes ADH1B e ADH1C}

As frequências alélicas dos polimorfismos $\mathrm{Arg} 48 \mathrm{His}$, Arg370Cys, Arg272GIn e lle350Val foram avaliadas comparativamente nas amostras das populações dos diferentes continentes a partir da pesquisa dos dados disponíveis on-line do projeto Ensembl Genome Browser 92 (https://www.ensembl.org/index.html) ${ }^{63}$. A Tabela 5 apresenta os principais resultados obtidos.

Tabela 5: Avaliação estatística dos dados disponíveis no projeto Ensembl genomas para as frequências dos polimorfismos Arg48His/Arg370Cys no gene ADH1B e lle350Val/Arg272Gln no gene ADH1C

\begin{tabular}{|c|c|c|c|c|}
\hline \multirow{2}{*}{ Polimorfismos } & \multirow{2}{*}{ Continentes } & \multirow{2}{*}{ Alelos* \% } & \multicolumn{2}{|r|}{ Valor de $p^{* *}$} \\
\hline & & & Geral & Específico \\
\hline \multicolumn{5}{|l|}{ Arg48His (C > T) } \\
\hline \multirow[t]{5}{*}{ (rs1229984) } & 1. Africano & $C=99,80 \mathrm{~T}=0,02$ & & $(1 \times 2=<0,01)(1 \times 3=<0,01)$ \\
\hline & 2. Americano & $C=94,20 T=5,80$ & & $(1 \times 4=<0,01)(1 \times 5=<0,01)$ \\
\hline & 3. Leste Asiático & $C=30,30 T=69,70$ & $<0,01$ & $(2 \times 3=<0,01)(2 \times 4=<0,01)$ \\
\hline & 4. Europeu & $C=97,10 \mathrm{~T}=2,90$ & & $(2 \times 5=<0,01)(3 \times 4=<0,01)$ \\
\hline & 5. Sul Asiático & $C=98,00 T=2,00$ & & $(3 \times 5=<0,01)(4 \times 5=>0,05)$ \\
\hline \multicolumn{5}{|l|}{ Arg370Cys (G > A) } \\
\hline \multirow[t]{5}{*}{ (rs2066702) } & 1. Africano & $G=80,90 A=19,10$ & & $(1 \times 2=<0,01)(1 \times 3=<0,01)$ \\
\hline & 2. Americano & $G=98,00 A=2,00$ & & $(1 \times 4=<0,01)(1 \times 5=<0,01)$ \\
\hline & 3. Leste Asiático & $G=100$ & $<0,01$ & $(2 \times 3=<0,01)(2 \times 4=<0,01)$ \\
\hline & 4. Europeu & $G=100$ & & $(2 \times 5=<0,01)(3 \times 4=>0,05)$ \\
\hline & 5. Sul Asiático & $G=100$ & & $(3 \times 5=>0,05)(4 \times 5=>0,05)$ \\
\hline \multicolumn{5}{|l|}{$\operatorname{Arg} 272 G \ln (C>T)$} \\
\hline \multirow[t]{5}{*}{ (rs1693482) } & 1. Africano & $C=90,00 T=10,00$ & & $(1 \times 2=<0,01)(1 \times 3=>0,05)$ \\
\hline & 2. Americano & $\mathrm{C}=72,90 \mathrm{~T}=27,10$ & & $(1 \times 4=<0,01)(1 \times 5=<0,01)$ \\
\hline & 3. Leste Asiático & $C=92,40 \mathrm{~T}=7,60$ & $<0,01$ & $(2 \times 3=<0,01)(2 \times 4=<0,01)$ \\
\hline & 4. Europeu & $C=59,50 T=40,50$ & & $(2 \times 5=>0,05)(3 \times 4=<0,01)$ \\
\hline & 5. Sul Asiático & $C=72,50 T=27,50$ & & $(3 \times 5=<0,01)(4 \times 5=<0,01)$ \\
\hline \multicolumn{5}{|l|}{ Ile350Val $(T>C)$} \\
\hline \multirow[t]{5}{*}{$($ rs698) } & 1. Africano & $\mathrm{T}=90,10 \mathrm{C}=9,90$ & & $(1 \times 2=<0,01)(1 \times 3=>0,05)$ \\
\hline & 2. Americano & $\mathrm{T}=72,90 \mathrm{C}=27,10$ & & $(1 \times 4=<0,01)(1 \times 5=<0,01)$ \\
\hline & 3. Leste Asiático & $\mathrm{T}=92,40 \mathrm{C}=7,60$ & $<0,01$ & $(2 \times 3=<0,01)(2 \times 4=<0,01)$ \\
\hline & 4. Europeu & $\mathrm{T}=59,50 \mathrm{C}=40,50$ & & $(2 \times 5=>0,05)(3 \times 4=<0,01)$ \\
\hline & 5. Sul Asiático & $\mathrm{T}=72,40 \mathrm{C}=27,60$ & & $(3 \times 5=<0,01)(4 \times 5=<0,01)$ \\
\hline
\end{tabular}

*As frequências alélicas foram obtidas do projeto Ensemb/ Genome Browser 92 (https://www.ensembl.org/index.html) ${ }^{63} \mathrm{e}$ a análise estatística foi conduzida no presente estudo; ${ }^{*}$ Valores de $p$ foram estimados pelos testes qui-quadrado de Pearson ou exato de Fisher conforme apropriado. Em negrito estão os valores de $p$ não significativos 
Em geral, o alelo variante (T, His) do polimorfismo Arg48His apresentou frequência reduzida nas amostras oriundas dos continentes americano, europeu, sul-asiático e africano $(5,8 \%, 2,9 \%, 2,0 \%$ e $0,02 \%$, respectivamente). Em contrapartida, foi frequente em amostras do Leste Asiático (69,7\%). Houve diferença estatística entre as frequências alélicas do polimorfismo Arg48His $(p<0,01)$, quando comparados todos os continentes, e quando avaliados por comparações individuais as frequências foram similares apenas entre europeus e sul-asiáticos $(p>0,05)$.

O polimorfismo Arg370Cys não foi detectado em amostras provenientes da Europa, leste e sul da Ásia, sendo considerado monomórfico nas populações destas regiões. No entanto, este alelo apresentou uma relativa frequência em populações da África $(19,1 \%)$ e está presente em $2,0 \%$ dos indivíduos oriundos do continente americano. Houve diferença estatística entre as frequências alélicas do polimorfismo Arg370Cys $(p<0,01)$, quando comparados todos os continentes, e quando avaliados por comparações individuais as frequências foram similares apenas entre leste e sul-asiáticos, leste-asiáticos e europeus e por fim entre sul-asiáticos e europeus ( $p>0,05$ nas três comparações) (Tabela 5).

O polimorfismo Arg272GIn apresentou frequências de $40,5 \%, 27,5 \%, 27,1 \%, 10,0 \%$ e $7,6 \%$, em amostras provenientes dos continentes europeu, sul-asiático, americano, africano e leste-asiático. Houve diferença estatística entre as frequências alélicas do polimorfismo $\operatorname{Arg} 272 \mathrm{G} \ln (p<0,01)$, quando comparados todos os continentes, e quando avaliados por comparações individuais as frequências foram similares apenas entre africanos e leste-asiáticos, e entre americanos e sul-asiáticos ( $p>0,05$ nas duas comparações) (Tabela 5).

O polimorfismo lle $350 \mathrm{Val}$ apresentou frequências de $40,5 \%, 27,6 \%, 27,1 \%, 9,9 \%$ e $7,6 \%$, em amostras provenientes dos continentes europeu, sul-asiático, americano, africano e leste-asiático. Houve diferença estatística entre as frequências alélicas do polimorfismo Ile350Val $(p<0,01)$, quando comparados todos os continentes, e quando avaliados por comparações individuais as frequências foram similares apenas entre africanos e leste-asiáticos, e entre americanos e sul-asiáticos ( $p>0,05$ nas duas comparações) (Tabela 5).

\section{DISCUSSÃO}

Os avanços científicos recentes no estudo de genomas estão possibilitando o entendimento de aspectos genéticos em diferentes níveis no organismo humano e relacionados ao desenvolvimento do TUA. Polimorfismos em genes associados a comportamentos impulsivos, desinibição e compulsividade foram relacionados previamente ao TUA ${ }^{13-19}$. Entretanto, diversos estudos relatam a importância de uma transformação bioquímica essencial na metabolização do álcool e que é fundamental na eliminação da molécula do organismo para evitar efeitos adversos mais severos e também o TUA ${ }^{20,21}$. O álcool etílico é convertido para acetaldeído pela ADH e depois para acetato pela enzima acetaldeído desidrogenase no fígado ${ }^{12,14,21}$. Variações nas atividades destas enzimas ocorrem em diferentes populações humanas, permitindo que ocorram diferentes concentrações de acetaldeído no fígado ${ }^{14-16,21}$.

Os achados nos genes ADH têm confirmado a diversidade de variações enzimáticas. Inicialmente, foram demonstradas que as variações alélicas no cromossomo 4 estão associadas ao TUA. Estas evidências foram consolidadas a partir de análises de base genética familiar ${ }^{16-18}$. Posteriormente observou-se que a região 4q21-23 possui os genes que codificam as diferentes subunidades da $\mathrm{ADH}^{16-18}$. A evolução das tecnologias de análises moleculares possibilitou a avaliação de genomas completos e estudos de GWAS trouxeram evidências científicas mais definitivas do envolvimento de alelos dos genes ADH no TUA ${ }^{10,12,18,22,23,29,30}$.

Esses SNPs nos genes $A D H$ são alvos de estudos em diferentes populações ${ }^{10,12,15}$. Os achados mais frequentemente reproduzidos são os que analisaram os genes $A D H 1 B$ e $A D H 1 C$. A associação do gene $A D H 1 B$ com o TUA foi demonstrada em estudos que avaliaram principalmente amostras de euroamericanos, afrodescendentes, europeus, asiáticos e nativo-americanos (Tabelas 1 e 2). Com relação às avaliações da relação entre o gene $A D H 1 C$ e o TUA, estudos têm sido realizados principalmente em amostras que incluíram europeus (Tabelas 3 e 4). Neste aspecto, no estudo de Tóth et al. $(2011)^{55}$, os polimorfismos lle350Val e Arg272GIn foram associados ao TUA. Estes polimorfismos são conhecidos também por apresentarem um desequilíbrio de ligação praticamente completo, sendo co-herdados em aproximadamente $99 \%$ das vezes, o que explica as frequências similares dos alelos variantes em diferentes populações ${ }^{8,14,39,55}$, como pode ser observado na Tabela 5.

No cenário brasileiro, há poucas pesquisas que contemplam o conhecimento das relações entre os polimorfismos nos genes $A D H 1 B, A D H 1 C$ e o TUA. Muito embora existam estudos que avaliaram polimorfismos nos genes $A D H$ associando ao câncer aerodigestivo ${ }^{64-67}$. Além destes, Guindalini et al. $(2005)^{68}$ encontraram associações $(\mathrm{OR}=2,9$; IC95\%: $1,89-4,73 ; p<0,01)$ entre SNPs localizados upstream (-75pb e -159pb) ao gene ADH4 com a dependência alcoólica. Já Rebello et al. $(2011)^{57}$ 
investigaram o polimorfismo lle350Val no gene $A D H 1 C$, porém não encontraram associações $(p>0,05)$ com o TUA. Carvalho et al. $(2013)^{38}$ demonstraram que o polimorfismo $\mathrm{Arg} 48 \mathrm{His}$ foi associado com a proteção para o TUA $(\mathrm{OR}=0,45$; IC95\%: 0,21 - 0,95; $p=0,03$ ), mas lle350Val não apresentou associação significativa $(p>0,05)$. Adicionalmente, Wolf et al. (2017) ${ }^{39}$ avaliaram 365 amostras de pacientes HIV positivos (121 casos e 244 controles) quanto aos polimorfismos Arg48His, Arg370Cys, Arg272GIn e lle350Val, sendo detectada uma associação significativa entre Arg48His e a proteção para o TUA (OR $=0,36$; IC95\%: 0,14-0,90; $p=0,03$ ). Não foram encontradas quaisquer associações com os demais polimorfismos estudados $(p>0,05)$.

É importante destacar que há indícios de diferenças entre as frequências alélicas dos polimorfismos nos genes $A D H$ entre as regiões do Brasi ${ }^{69,70}$. Porém, falta representatividade e poder estatístico das amostras avaliadas nestes estudos. Muito provavelmente o fato de que há uma ampla variedade de etnias que compõem a população brasileira contribui de forma importante para os diferentes perfis de frequências alélicas dos polimorfismos nos genes $A D H$, e consequentemente a interação entre as características genéticas e ambientais podem determinar influencias específicas para o desfecho do TUA, dependendo da região do país. Contudo, dado o nosso conhecimento, não há estudos disponíveis que avaliaram amostras representativas de todo o Brasil, com poder estatístico, e que analisaram comparativamente as frequências dos polimorfismos dos genes $A D H 1 B e$ $A D H 1 C$ entre as diferentes regiões. Estudos futuros que visem abordar este tema serão importantes para o melhor entendimento da diversidade dos genes $A D H$ no Brasil, e como este processo pode afetar o TUA.

Os estudos do tipo GWAS realizam uma varredura nos genomas dos indivíduos avaliados, e determinam os polimorfismos associados a um dado desfecho. Há vários GWAS disponíveis na literatura que identificaram polimorfismos nos genes $A D H$ e que foram estatisticamente associados ao TUA ${ }^{12,17,20,22-26}$. A partir deste conhecimento, a identificação de indivíduos que apresentem predisposição genética ao TUA pode ser uma perspectiva para que se evite o estabelecimento deste transtorno, e tal conduta poderá ser empregada no futuro. Além disso, avaliações de polimorfismos nos genes $A D H$ e das atividades metabólicas das isoenzimas $A D H$, poderão auxiliar no manejo terapêutico do TUA, visto que há fármacos que atuam modulando a metabolização do álcool, como por exemplo, o dissulfiram ${ }^{71}$. Nesse sentido, estudos que avaliem questões farmacogenéticas poderão ser importantes para um melhor manejo terapêutico dos pacientes com TUA.

O presente estudo possui como principal limitação o fato de não sistematizar os resultados dos diferentes estudos disponíveis na literatura. Além disso, os desfechos dos problemas relacionados ao uso do álcool dos estudos selecionados para revisão não foram padronizados, visto que alguns avaliaram a dependência alcoólica, enquanto outros avaliaram o consumo abusivo e/ou reações fisiológicas ao álcool. Outro fator importante a ser destacado é o método diagnóstico empregado, aspecto não padronizado entre os estudos. Nesse sentido, foram empregadas diferentes formas de detectar transtornos relacionados ao uso do álcool, como o DSM (do inglês, Diagnostic and Statistical Manual of Mental Disorders), o CAGE (acrônimo inglês para Cutting down, Annoyance by criticism, Guilty feeling, and Eye-openers) e o AUDIT (do inglês, Alcohol Use Disorder Identification Test). De qualquer forma, o diagnóstico predominante nos estudos selecionados foi a dependência alcoólica, isso nos permite uma visão geral da influência dos polimorfismos nos genes $A D H 1 B$ e $A D H 1 C$ no TUA. Apesar das limitações deste estudo, os dados levantados serão úteis para compreensão de uma importante questão que afeta o TUA, o qual é um transtorno responsável por gerar agravos sociais devastadores. Estudos futuros que avaliem os polimorfismos nos genes $A D H$ serão fundamentais para o preenchimento das lacunas científicas que ainda existem entre a genética e o TUA. Além disso, os avanços tecnológicos observados atualmente podem contribuir para o estabelecimento de intervenções em indivíduos predispostos geneticamente ao TUA.

\section{CONCLUSÕES}

Este estudo reuniu os dados da literatura sobre as contribuições dos principais polimorfismos nos genes de metabolização do álcool com relação ao TUA. Os polimorfismos Arg48His e Arg370Cys são os que apresentam maiores efeitos para a proteção do TUA, e suas frequências são caracteristicamente elevadas na Ásia e na África, respectivamente. Apesar disso, estudos já demonstraram que Arg48His está associado com a proteção ao TUA no Brasil. Por outro lado, os polimorfismos Arg272GIn e lle350Val, os quais são mais frequentes nos continentes europeu e americano, atuam predispondo ao TUA. Finalmente, este estudo abordou um tema de grande importância para a saúde pública, visto que o avanço técnico-científico observado na atualidade poderá gerar perspectivas para a detecção destes polimorfismos, podendo assim ser uma ferramenta auxiliar para o manejo dos indicadores epidemiológicos do TUA. 


\section{REFERÊNCIAS}

1. Connor JP, Haber PS, Hall WD. Alcohol use disorder. Lancet. 2016;387(10022):988-98.

2. McGovern PE. Uncorking the past: the quest for wine, beer, and other alcoholic beverages. Berkley (CA): University of California Press; 2009 [citado 2020 Fev 13]. Disponível em: http://www.upenn.edu/gazette/0110/ PennGaz0110_feature1.pdf

3. World Health Organization [Internet]. Genebra; 2016 [citado 2020 Fev 13]. Global health observatory (GHO) data; [aproximadamente 1 tela]. Disponível em: http://www.who.int/gho/alcohol/en/

4. World Health Organization [Internet]. Genebra; 2015 [citado 2020 Fev 13]. Alcohol [aproximadamente 10 telas]. Disponível em: http://www.who.int/ mediacentre/factsheets/fs349/en/

5. Laranjeira R, Pinsky I, Zaleski M, Caetano R. I Levantamento nacional sobre os padrões de consumo de álcool na população brasileira. Brasília: Secretaria Nacional Antidrogas; 2007 [citado 2020 Fev 13]. Disponível em: http:// bvsms.saude.gov.br/bvs/publicacoes/ relatorio_padroes_consumo_alcool.pdf

6. Cisa [Internet]. São Paulo; 2017 [citado 2020 Fev 17]. Dados epidemiológicos sobre o uso do álcool no Brasil; [aproximadamente 3 telas]. Disponível em: http://www.cisa.org. br/artigo/11/dados-epidemiologicossobre-uso-alcool-no.php

7. WHO. Global status report on alcohol and health 2014. Genebra; 2014.

8. Edenberg HJ, Foroud T. Genetics and alcoholism. Nat Rev Gastroenterol Hepatol. 2013;10(8):487-94.

9. Wall TL, Luczak SE, Hiller-Sturmhöfel S. Biology, genetics, and environment: underlying factors influencing alcohol metabolism. Alcohol Res. 2016;38(1):59-68.

10. Bierut LJ. Genetic vulnerability and susceptibility to substance dependence. Neuron. 2011;69(4):618-27.

11. Kimura M, Higuchi S. Genetics of alcohol dependence. Psychiatry Clin Neurosci. 2011;65:213-25.
12. Tawa EA, Hall SD, Lohoff FW. Overview of the genetics of alcohol use disorder. Alcohol. 2016;51(5):507-14.

13. Edenberg HJ. Genetics of alcohol use disorders. In: Miller PM, editor. Biological research on addiction. London: Elsevier; 2013. p. 500-8.

14. Edenberg HJ. The genetics of alcohol metabolism: role of alcohol dehydrogenase and aldehyde dehydrogenase variants. Alcohol Res Health. 2007;30(1):5-13.

15. Hurley TD, Edenberg HJ. Genes encoding enzymes involved in ethanol metabolism. Alcohol Res. 2012;34(3):339-44.

16. Wang JC, Kapoor M, Goate AM. The genetics of substance dependence. Annu Rev Genomics Hum Genet. 2012;13:241-61.

17. Samochowiec J, Samochowiec A, Puls I, Bienkowski P, Schott BH. Genetics of alcohol dependence: a review of clinical studies. Neuropsychobiology. 2014;70(2):77-94.

18. Stickel F, Moreno C, Hampe $\mathrm{J}$, Morgan MY. The genetics of alcohol dependence and alcoholrelated liver disease. $J$ Hepatol. 2017;66(1):195-211.

19. Yu C, McClellan J. Genetics of substance use disorders. Child Adolesc Psychiatr Clin N Am. 2016;25(3):377-85.

20. Polimanti R, Gelernter J. ADH1B: from alcoholism, natural selection, and cancer to the human phenome. Am J Med Genet B Neuropsychiatr Genet. 2018;177(2):113-25.

21. Zakhari, S. Overview: how is alcohol metabolized by the body? Alcohol Res Health. 2006;29(4):245-54.

22. Treutlein J, Rietschel M. Genomewide association studies of alcohol dependence and substance use disorders. Curr Psychiatry Rep. 2011;13(2):147-55.

23. Gelernter J, Kranzler HR, Sherva R, Almasy L, Koesterer R, Smith AH, et al. Genome-wide association study of alcohol dependence: significant findings in African- and EuropeanAmericans including novel risk loci. Mol Psychiatry. 2014;19(1):41-9.
24. Hart AB, Lynch KG, Farrer L, Gelernter J, Kranzler HR. Which alcohol use disorder criteria contribute to the association of ADH1B with alcohol dependence? Addict Biol. 2016;21(4):924-38.

25. Xu K, Kranzler HR, Sherva R, Sartor CE, Almasy L, Koesterer R, et al. Genomewide association study for maximum number of alcoholic drinks in European Americans and african americans. Alcohol Clin Exp Res. 2015;39(7):1137-47.

26. Park BL, Kim JW, Cheong HS, Kim $\mathrm{LH}$, Lee BC, Seo $\mathrm{CH}$, et al. Extended genetic effects of $A D H$ cluster genes on the risk of alcohol dependence: from GWAS to replication. Hum Genet. 2013;132(6):657-68.

27. Luo X, Kranzler HR, Suo L, Wang S, Stork NJ, Gelernter J. Diplotype trend regression analysis of the ADH gene cluster and the ALDH2 gene: multiple significant associations with alcohol dependence. Am J Hum Genet. 2006;78(6):973-87.

28. Tolstrup JS, Nordestgaard BG, Rasmussen S, Tybjaerg-Hansen A, Grønbaek M. Alcoholism and alcohol drinking habits predicted from alcohol dehydrogenase genes. Pharmacogenomics J. 2008;8(3):220-7.

29. Sherva R, Rice JP, Neuman RJ, Rochberg N, Saccone NL, Bierut LJ. Associations and interactions between SNPs in the alcohol metabolizing genes and alcoholism phenotypes in European Americans. Alcohol Clin Exp Res. 2009;33(5):848-57.

30. Bierut LJ, Goate AM, Breslau N, Johnson EO, Bertelsen S, Fox L, et al. ADH1B is associated with alcohol dependence and alcohol consumption in populations of European and African ancestry. Mol Psychiatry. 2012;17(4):445-50.

31. Ehlers CL, Liang T, Gizer IR. ADH and ALDH polymorphisms and alcohol dependence in Mexican and Native Americans. Am J Drug Alcohol Abuse. 2012;38(5):389-94.

32. Borràs $E$, Coutelle $C$, Rosell $A$, Fernández-Muixi $F$, Broch $M$, Crosas $B$, et al. Genetic polymorphism of alcohol dehydrogenase in Europeans: the $\mathrm{ADH} 2{ }^{*} 2$ allele decreases 
the risk for alcoholism and is associated with ADH3*1. Hepatology. 2000;31(4):984-9.

33. Cichoż-Lach H, Celiński K, Wojcierowski J, Słomka M, Lis E. Genetic polymorphism of alcoholmetabolizing enzyme and alcohol dependence in Polish men. Braz J Med Biol Res. 2010;43(3):257-61.

34. Linneberg A, Gonzalez-Quintela A, Vidal C, Jørgensen T, Fenger $M$, Hansen T, et al. Genetic determinants of both ethanol and acetaldehyde metabolism influence alcohol hypersensitivity and drinking behaviour among Scandinavians. Clin Exp Allergy. 2010;40(1):123-30.

35. Toth R, Pocsai Z, Fiatal S, Szeles G, Kardos L, Petrovski B, et al. ADH1B*2 allele is protective against alcoholism but not chronic liver disease in the Hungarian population. Addiction. 2010;105(5):891-6.

36. Hubacek JA, Pikhart H, Peasey A, Kubinova R, Bobak M. ADH1B polymorphism, alcohol consumption and binge drinking in Slavic Caucasians: results from the Czech HAPIEE study. Alcohol Clin Exp Res. 2012;36(5):900-5.

37. Way M, McQuillin A, Saini J, Ruparelia K, Lydall GJ, Guerrini I, et al. Genetic variants in or near ADH1B and $\mathrm{ADH} 1 \mathrm{C}$ affect susceptibility to alcohol dependence in a British and Irish population. Addict Biol. 2015;20(3):594-604.

38. Carvalho MM. Análise de polimorfismos genéticos dos genes $A D H 1 B, A D H 1 C$ e CYP2E1 de metabolização do álcool em uma população de alcoolistas do nordeste do brasil [dissertação]. Teresina: Universidade Federal do Piauí; 2013. $124 \mathrm{p}$.

39. Wolf JM, Simon D, Béria JU, Tietzmann DC, Stein AT, Lunge VR. Analysis of the association of nonsynonymous polymorphisms in $\mathrm{ADH}$ genes with hazardous drinking in HIV-1 positive individuals. Alcohol Clin Exp Res. 2017;41(11):1866-74.

40. Chen WJ, Loh EW, Hsu YP, Chen CC, Yu JM, Cheng AT. Alcoholmetabolising genes and alcoholism among Taiwanese Han men: independent effect of ADH2, ADH3 and ALDH2. Br J Psychiatry. 1996;168(6):762-7.
41. Tanaka F, Shiratori Y, Yokosuka O, Imazeki F, Tsukada Y, Omata M. High incidence of ADH2*1/ ALDH2*1 genes among Japanese alcohol dependents and patients with alcoholic liver disease. Hepatology. 1996;23(2):234-9.

42. Li D, Zhao H, Gelernter J. Strong association of the alcohol dehydrogenase $1 \mathrm{~B}$ gene (ADH1B) with alcohol dependence and alcoholinduced medical diseases. Biol Psychiatry. 2011;70(6):504-12.

43. Meyers JL, Shmulewitz D, Aharonovich E, Waxman R, Frisch A, Weizman A, et al. Alcohol metabolizing genes and alcohol phenotypes in an Israeli household sample. Alcohol Clin Exp Res. 2013;37(11):1872-81.

44. Kilcoyne B, Shmulewitz D, Meyers JL, Aharonovich E, Greenstein E, Frisch $A$, et al. Alcohol consumption mediates the relationship between $A D H 1 B$ and DSM-IV alcohol use disorder and criteria. J Stud Alcohol and Drugs Suppl. 2014;75(4):635-42.

45. Al-Tu'ma FJ, El-Yassin HD, Shammam $\mathrm{KH}$. Alcohol dehydrogenase genotype $(\mathrm{ADH} 2$ and $\mathrm{ADH} 3)$ in alcoholism of Iraqi people. J Cont Med Sci. 2015;1(2):9-13.

46. Macgregor S, Lind PA, Bucholz KK, Hansell NK, Madden PA, Richter $\mathrm{MM}$, et al. Associations of ADH and ALDH2 gene variation with self report alcohol reactions, consumption and dependence: an integrated analysis. Hum Mol Genet. 2009;18(3):580-93.

47. Ehlers CL, Carr L, Betancourt M, Montane-Jaime K. Association of the $\mathrm{ADH} 2 * 3$ allele with greater alcohol expectancies in African-American young adults. J Stud Alcohol. 2003;64(2):176-81.

48. Edenberg $\mathrm{HJ}$, Xuei $X$, Chen $\mathrm{HJ}$, Tian H, Wetherill LF, Dick DM, et al. Association of alcohol dehydrogenase genes with alcohol dependence: a comprehensive analysis. Hum $\mathrm{Mol}$ Genet. 2006;15(9):1539-49.

49. McCarthy DM, Pedersen SL, Lobos EA, Todd RD, Wall TL. ADH1B*3 and response to alcohol in African Americans. Alcohol Clin Exp Res. 2010;34(7):1274-81.

50. Wall TL, Carr LG, Ehlers CL. Protective association of genetic variation in alcohol dehydrogenase with alcohol dependence in Native American Mission Indians. Am J Psychiatry. 2003;160(1):41-6.

51. Gizer IR, Edenberg HJ, Gilder DA, Wilhelmsen KC, Ehlers CL. Association of alcohol dehydrogenase genes with alcohol-related phenotypes in a Native American community sample. Alcohol Clin Exp Res. 2011;35(11):2008-18.

52. Montane-Jaime LK, Shafe S, Liang T, Wills DN, Berg GI, Ehlers CL. Subjective response to alcohol and $\mathrm{ADH}$ polymorphisms in a select sample of young adult male East Indians and Africans in Trinidad and Tobago. J Stud Alcohol Drugs. 2014;75(5):827-38.

53. Kuo PH, Kalsi G, Prescott CA, Hodgkinson CA, Goldman D, van den Oord EJ, et al. Association of $\mathrm{ADH}$ and $\mathrm{ALDH}$ genes with alcohol dependence in the Irish Affected Sib Pair Study of alcohol dependence (IASPSAD) sample. Alcohol Clin Exp Res. 2008;32(5):785-95.

54. Martínez C, Galván S, Garcia-Martin E, Ramos MI, Gutiérrez-Martín Y, Agúndez JA. Variability in ethanol biodisposition in whites is modulated by polymorphisms in the ADH1B and $\mathrm{ADH} 1 \mathrm{C}$ genes. Hepatology. 2010;51(2):491-500.

55. Tóth R, Fiatal S, Petrovski B, McKee $\mathrm{M}$, Adány R. Combined effect of ADH1B RS1229984, RS2066702 and ADH1C RS1693482/RS698 alleles on alcoholism and chronic liver diseases. Dis Markers. 2011;31(5):267-77.

56. Kortunay S, Köseler A, Ozdemir F, Atalay EÖ. Association of a genetic polymorphism of the alcoholmetabolizing enzyme ADH1C with alcohol dependence: results of a case-control study. Eur Addict Res. 2012;18(4):161-6.

57. Ayhan Y, Gürel ŞC, Karaca Ö, Zoto T, Hayran M, Babaoğlu M, et al. Association between ADH1C and ALDH2 polymorphisms and alcoholism in a Turkish sample. Nord $\mathrm{J}$ Psychiatry. 2015;69(3):233-9.

58. Chen WJ, Loh EW, Hsu YP, Chen CC, Yu JM, Cheng AT. Alcoholmetabolising genes and alcoholism among Taiwanese Han men: independent effect of ADH2, ADH3 and ALDH2. Br J Psychiatry. 1996;168(6):762-7. 
59. Bjerregaard P, Mikkelsen SS, Becker U, Hansen T, Tolstrup JS. Genetic variation in alcohol metabolizing enzymes among Inuit and its relation to drinking patterns. Drug Alcohol Depend. 2014;144:239-44.

60. Montane-Jaime K, Moore S, Shafe S, Joseph R, Crooks H, Carr L, Ehlers $\mathrm{CL} . \mathrm{ADH} 1 \mathrm{C}^{\star} 2$ allele is associated with alcohol dependence and elevated liver enzymes in Trinidad and Tobago. Alcohol. 2006;39(2):81-6.

61. Rebello AS, Moura-Neto R, Carvalho MGC. Association study of the lle349val polymorphism of the gene ADH1C and alcohol dependence. $J$ Bras Psiquiatr. 2011;60(1):7-10.

62. Méndez C, Rey M. Characterization of polymorphisms of genes ADH2, $\mathrm{ADH} 3, \mathrm{ALDH} 2$ and CYP2E1 and relationship to the alcoholism in a Colombian population. Colomb Med (Cali). 2015;46(4):176-82.

63. Ensembl Genome Browser [Internet]; 2017 [citado 2020 Fev 17]. 1000 Genomes Project Phase 3 Allele
Frequencies; [aproximadamente 1 tela]. Disponível em: http://www. ensembl.org/index.html

64. Nishimoto IN, Pinheiro NA, Rogatto SR, Carvalho AL, Moura RP, Caballero $\mathrm{OL}$, et al. Alcohol dehydrogenase 3 genotype as a risk factor for upper aerodigestive tract cancers. Arch Otolaryngol Head Neck Surg. 2004;130(1):78-82.

65. Hashibe M, McKay JD, Curado MP, Oliveira JC, Koifman S, Koifman R, et al. Multiple $\mathrm{ADH}$ genes are associated with upper aerodigestive cancers. Nat Genet. 2008;40(6):707-9.

66. Garcia SM, Curioni OA, Carvalho MB, Gattás GJ. Polymorphisms in alcohol metabolizing genes and the risk of head and neck cancer in a Brazilian population. Alcohol. 2010;45(1):6-12

67. Takamori JT. Avaliação de polimorfismos em genes de metabolismo do etanol e gene de reparo do DNA em pacientes portadores de câncer de boca [dissertação]. São Paulo: Faculdade de Medicina de São Paulo; 2012. 74 p.
68. Guindalini C, Scivoletto S, Ferreira RG, Breen G, Zilberman M, Peluso MA, Zatz M. Association of genetic variants in alcohol dehydrogenase 4 with alcohol dependence in Brazilian patients. Am J Psychiatry. 2005;162(5):1005-7.

69. Alfred [Internet]; [citado 2020 Fev 17]. The ALlele FREquency Database; [aproximadamente 2 telas]. Disponível em: http://alfred.med.yale.edu/alfred/ index.asp

70. Hashibe M, McKay JD, Curado MP, Oliveira JC, Koifman S, Koifman R, et al. Multiple ADH genes are associated with upper aerodigestive cancers. Nat Genet. 2008;40(6):707-9.

71. Emea. Guideline on the development of medicinal products for the treatment of alcohol dependence. Londres; 2010 [citado 2020 Fev 17]. Disponível em: http://www.ema.europa.eu/docs/ en_GB/document_library/Scientific_ guideline/2010/03/WC500074898.pdf

Recebido: 20 out, 2019 Aceito: 10 jan, 2020 